\section{A novel scalar tracking method for optimising film cooling systems}

\author{
Mitra Thomas and Thomas Povey
}

Proc IMechE Part A:

$J$ Power and Energy

$0(0) \mathrm{I}-13$

(C) IMechE 2015

Reprints and permissions:

sagepub.co.uk/journalsPermissions.nav DOI: 10.1 I77/09576509|5605944

pia.sagepub.com

\begin{abstract}
The purpose of this paper is to outline a novel passive-scalar tracking method for computational fluid dynamics optimisation studies. An example of its utility is in isolating the contributions of individual film cooling holes to overall cooling effectiveness on a heavily film cooled component. To isolate individual cooling contributions, passive (not a physical property of the flow, and therefore non-interacting) scalar variables are associated with the flow at each cooling hole outlet, with the scalar variable diffusivity set equal to the effective turbulent thermal diffusivity to replicate the mixing behaviour of the thermal field. The scalar tracking method is demonstrated by application to the optimisation of a nozzle guide vane endwall film cooling system, allowing a highly optimised system to be designed in three to five computational fluid dynamics simulations, orders of magnitude faster than optimisations performed using automated design space exploration. The test case for the method is an improved design of platform cooling system in which a relatively small quantity of high-momentum coolant is injected upstream of the vanes to reduce the total pressure deficit in near-wall region, followed by additional film cooling in low Mach number regions deeper into the passage. The optimised design uses half the coolant mass flow of a baseline design, while maintaining similar cooling effectiveness levels in critical regions.
\end{abstract}

\title{
Keywords
}

Turbine blade cooling, turbine heat transfer, turbomachinery aerodynamics

Date received: 20 July 20I4; accepted: 14 July 2015

\section{Introduction}

Film cooling involves releasing coolant flow through holes and slots in a turbine component to form a protective film. Film cooling allows the turbine to operate at higher temperatures, resulting in a more efficient thermodynamic cycle and a more compact engine. However, coolant flow is drawn from the compressor leading to a performance penalty. Also, injection of coolant flow in the gas path leads to aerodynamic losses. Minimising the use of coolant flow is thus desirable in targeting the maximum possible efficiency.

This paper will discuss a new passive scalartracking method that will allow rapid computational optimisation of film cooling systems. This method will be demonstrated by application to the optimisation of a nozzle guide vane endwall film cooling system.

\section{Scalar tracking method}

\section{Theory}

The purpose of the passive-scalar tracking described in this paper is to allow the individual contributions of cooling holes to cooling effectiveness to be isolated.
Separation of individual contributions allows superposition to be used, allowing rapid convergence of optimised designs (typically within three to five computational fluid dynamics (CFD) simulations). The method requires a passive (non-physical property that does not interact with physical variables) scalar quantity to be associated with each cooling hole flow, allowing the concentration in the partially mixed out downstream flow to be determined by tracking individual scalar variables.

CFD solutions for the momentum and continuity equations are first obtained. The steady-state transport of the scalar, $\phi_{i}$, through the domain is modelled by the advection equation, shown below

$$
\frac{\partial\left(\rho u_{n} \phi_{i}\right)}{\partial x_{n}}=\Gamma \frac{\partial^{2} \phi_{i}}{\partial x_{n} \partial x_{n}}
$$

Department of Engineering Science, University of Oxford, Parks Road, Oxford, UK

\section{Corresponding author:}

Mitra Thomas, Department of Engineering Science, University of Oxford, Parks Road, Oxford, OXI 3PJ, UK.

Email: mitra.thomas@gmail.com 
Here, $\phi_{i}$ is the concentration of a scalar, indexed $i$, $\Gamma$ is the scalar diffusivity; $u_{n}$ is the velocity vector where $n$ is the coordinate index. To make the scalar reproduce the mixing behaviour of the thermal field, scalar diffusivity is set to effective turbulent thermal diffusivity, $k_{\text {eff }}$. That is $\Gamma=k_{\text {eff }}=\left(k+\left(\frac{c_{p} \mu_{T}}{\mathrm{Pr}_{\mathrm{t}}}\right)\right)$, where $k$ is the laminar thermal conductivity, $\mu_{T}$ is the eddy viscosity, and $\operatorname{Pr}_{t}$ is the turbulent Prandtl number.

The following boundary condition is set at the inlet to cooling hole $j$

$$
\begin{aligned}
& \phi_{i}=1, \text { for } j=i \\
& \phi_{i}=0, \text { for } j \neq i
\end{aligned}
$$

where the index $i$ denotes the scalar variable number and index $j$ denotes the cooling hole number. The boundary condition $\phi_{i}=0$ is set at the freestream inlet, for all $i$. A control volume analysis is now used to relate passive-scalar concentration, $\phi_{i}$, to coolant concentration (Figure 1).

Scalar concentration in the mixed condition is given by

$$
\phi_{i}=\frac{\dot{m}_{c, i} \times\left.\phi_{i}\right|_{c, i}+\dot{m}_{e} \times\left.\phi_{i}\right|_{\infty}}{\Sigma_{i} \dot{m}_{c, i}+\dot{m}_{e}}=\frac{\dot{m}_{c, i}}{\dot{m}_{m}}
$$

since the concentration of scalar $i$ from cooling hole $i$, $\left.\phi_{i}\right|_{c, i}=1$ (equation (2)), and the concentration of scalar $i$ in the entrained freestream flow, $\left.\phi_{i}\right|_{\infty}=0$.

The scalar concentration, $\phi_{i}$, is the contribution to coolant mass fraction from hole $i$. If the scalar concentrations were summed over all cooling holes, the net coolant mass fraction is obtained

$$
\sum_{i} \phi_{i}=\frac{\dot{m}_{c}}{\dot{m}_{m}}
$$

The continuity and energy equations are

$$
\dot{m}_{m}=\dot{m}_{c}+\dot{m}_{e}
$$

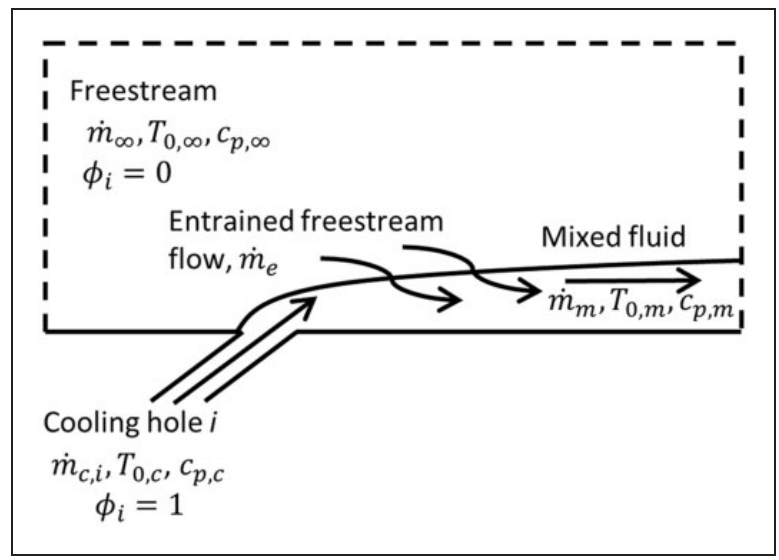

Figure I. Control volume analysis for scalar concentration.

$$
\dot{m}_{m} c_{p, m} T_{0, m}=\dot{m}_{e} c_{p, \infty} T_{0, \infty}+\dot{m}_{c} c_{p, c} T_{0, c}
$$

To demonstrate the simplest possible implementation of the scalar tracking method, the following assumptions are made: flow is incompressible, and $c_{p} \neq f(T)$. Improved formulations of the scalar tracking method with these assumptions relaxed are discussed in Appendix 2.

For $c_{p} \neq f(T)$, i.e. $c_{p, m}=c_{p, \infty}=c_{p, c}$, dividing equation (6) by $\dot{m}_{m}$, eliminating $\dot{m}_{e}$ using equation (5) and substituting $\sum \phi_{i}$ from equation (4), the following expression is obtained

$$
T_{0, m}=T_{0, \infty}-\left(\sum_{i} \phi_{i}\right)\left(T_{0, \infty}-T_{0, c}\right)
$$

In incompressible flow, the adiabatic wall temperature, $T_{a w}$, is equal to the near-wall mixed total temperature. Setting $T_{a w}=T_{0, m}$ in equation (7), the following expression is obtained

$$
\Sigma \phi_{i}=\frac{T_{0, \infty}-T_{a w}}{T_{0, \infty}-T_{0, c}}=\eta_{e f f}
$$

where $\eta_{\text {eff }}$ is the adiabatic cooling effectiveness. The sum of scalar concentration, $\Sigma \phi_{i}$, is thus a proxy for $\eta_{\text {eff. }}$ In particular, the contribution of an individual hole (of index $i$ ) to $\eta_{e f f}$ is given by $\phi_{i}$, the concentration of the scalar associated with the hole.

The incompressible definition of $\eta_{\text {eff }}$ is chosen to illustrate the simplest possible implementation of the new passive-scalar tracking method. In situations dominated by high Mach number flow, it is simpler to work with near-wall total temperature, $T_{0, m}$, as an optimisation target, rather than $\eta_{\text {eff }}$. This implementation is discussed in Appendix 2.

The variation of specific heat with temperature was neglected in this formulation. In a gas turbine engine, $c_{p}$ varies by about $10 \%$ over the range of temperatures between coolant and mainstream. An improved derivation accounting for this variation is provided in Appendix 2.

The effect of varying hole diameters is now considered. For small changes in the diameter of hole $i, D_{i}$, coolant flow rate, $\dot{m}_{c, i}$, varies as $D_{i}^{2}$. Thus the cooling effectiveness contribution can be scaled for small changes in hole diameter using the following equation

$$
\phi_{i, n e w}=\frac{D_{i, n e w}^{2}}{D_{i}^{2}} \phi_{i}
$$

where the suffix 'new' corresponds to the new diameter hole. $\eta_{\text {eff }}$ distributions can thus be estimated for cooling systems of different diameter distributions, but with holes at the same locations, using the 
Table I. Vane cascade geometrical details.

\begin{tabular}{ll}
\hline Vane geometry & \\
\hline Tangential chord length (at midspan) & $227 \mathrm{~mm}$ \\
Axial chord length (at midspan) & $110 \mathrm{~mm}$ \\
Inlet and outlet angle & $0^{\circ}$ and $74^{\circ}$ \\
Pitch/tangential chord & 1.0 \\
Inlet span/tangential chord & 1.2 \\
Outlet span/tangential chord & 1.0 \\
\hline
\end{tabular}

superposition equation

$$
\eta_{\text {eff }, \text { new }}=\sum_{i} \phi_{i, \text { new }}=\sum_{i} \frac{D_{i, \text { new }}^{2}}{D_{i}^{2}} \phi_{i}
$$

This offers a tool for optimisation of film cooling systems.

\section{Implementation of scalar tracking method in CFD}

CFD methods. The test cascade and CFD methods used are now discussed. A two-passage CFD domain was used to simulate flow through a test cascade. The geometry of the cascade is that used in a large-scale platform cooling test facility in Oxford University, ${ }^{1}$ which includes a linearised NGV geometry from a recent commercial engine. Geometric details of the cascade are provided in Table 1.

The CFD domains used are shown in Figure 2. Separate CFD domains were used to simulate casing (a) and hub (b) film cooling configurations to minimise cell count. Geometrical details for the baseline cooling hole configuration are given in Table 2.

Periodic boundary conditions are used on either side of the two-passage domain. The double-row film cooling hole pattern occupies the central 1.8 vane pitch lengths $(V)$ replicating the configuration in the test facility. Cooling holes are not included within $0.1 \mathrm{~V}$ of the periodic boundary to simplify mesh generation (results from the central passage are used for analysis).

The simulations described in this study were performed at test facility representative flow conditions. ${ }^{1}$ The boundary conditions used are given in Table 3 . Adiabatic conditions were prescribed on all wall surfaces.

Tetrahedral meshes were generated in ICEM-CFD. Flow solutions were obtained using the FLUENT solver. Mesh independence was judged by comparing $\eta_{\text {eff }}$ on lines on the endwall surface for solutions of increasing mesh sizes -0.3 million, 1.2 million, 1.7 million, 4.25 million, 8 million cells. The difference in $\eta_{\text {eff }}$ between the solution for 4.25 million and 8 million cells was less than $5 \%$. Meshes of approximate cell count of 8 million cells were thus used for all

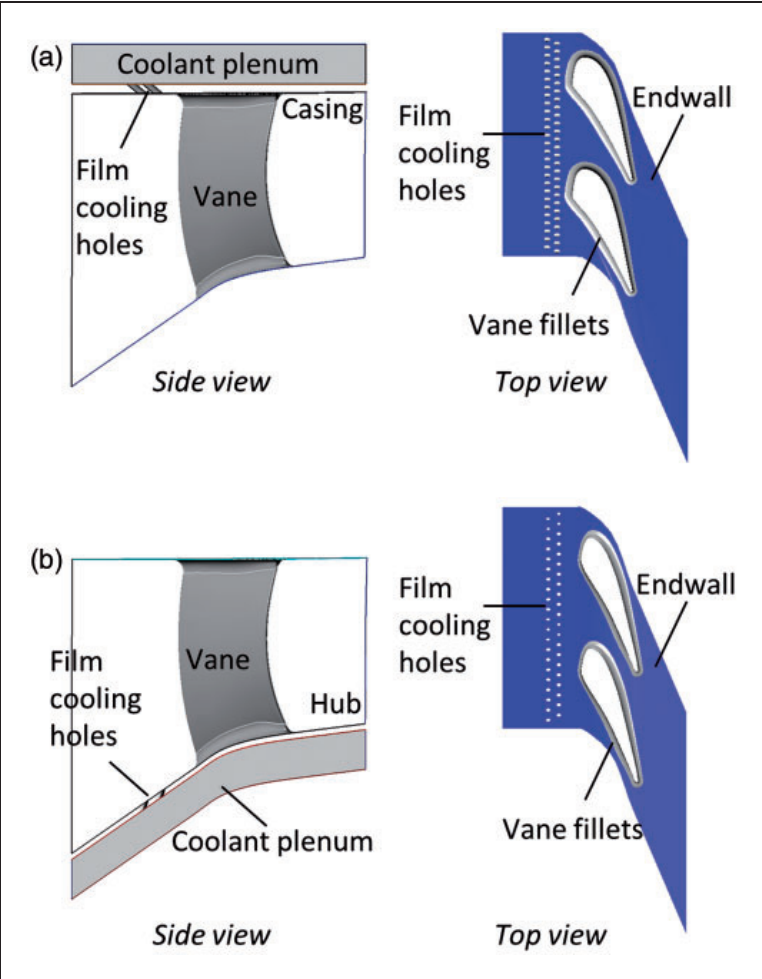

Figure 2. CFD model schematics: (a) casing endwall: CFD model; (b) hub endwall: CFD model.

Table 2. Geometrical details.

\begin{tabular}{lll}
\hline Baseline cooling hole geometry & & \\
\hline & Casing & Hub \\
\hline Number of rows & 2 & 2 \\
Diameter & $8 \mathrm{~mm}$ & $5.6 \mathrm{~mm}$ \\
Hole inclination to endwall & $40^{\circ}$ & $40^{\circ}$ \\
Hole pitch/diameter & 2.0 & 3.6 \\
Row axial spacing/diameter & 2.8 & 3.8 \\
Hole length/diameter & 3.8 & 6.5 \\
\hline
\end{tabular}

the solutions described in this paper. The mesh resolution on the cooled endwall is shown in Figure 3.

The realisable $k-\varepsilon$ model was used with standard wall functions, because of its ability to predict mixing, shear layers and boundary layer separation with reasonable accuracy. ${ }^{2}$ Mesh sizes were prescribed to ensure $30<\mathrm{y}^{+}<300$ on the endwall.

Thomas et al., ${ }^{1}$ describe experimental validation of the CFD methods presented in this study: experimental maps of endwall $\eta_{\text {eff }}$ obtained at a number of coolant to mainstream mass flux ratios, showing good agreement with CFD predictions.

Implementation in FLUENT and validation. The implementation of the passive-scalar tracking method in CFD is 
Table 3. CFD boundary conditions.

\begin{tabular}{ll}
\hline Mainstream conditions & $P_{0, \infty}=1024 \mathrm{~Pa}$ (gauge) \\
& $P_{\text {out }}=0 \mathrm{~Pa}$ (gauge) \\
& $T_{0, \infty}=313 \mathrm{~K}$ \\
& $T u=15 \%, I=0.22 \mathrm{~V}$ \\
& $R e$ (based on outlet \\
conditions $)=7.5 \times 10^{5}$ \\
& $(\mathrm{~V}=$ vane pitch) \\
& $T_{0, c}=293 \mathrm{~K}$ \\
Coolant conditions & Specified coolant mass flux, $\dot{m}_{c}$. \\
& $T u=15 \%, I=0.22 \mathrm{~V}$ \\
\hline
\end{tabular}

now described in relation to Figure 4. Surfaces were created at the hole inlets. Solutions for the continuity, momentum and energy equations were obtained on a CFD model with a coolant plenum (Figure 4(a)). Flow profiles were outputted from the hole inlet boundary surfaces (Figure 4(b)). These were values of $P_{0}, T_{0}, k, \varepsilon$, and unit vectors of velocity direction. The flow profiles were set as boundary conditions on a CFD model without a coolant plenum (Figure 4(c)) and CFD solutions were obtained. Postprocessing lines on the hub endwall are shown in Figure 4(d).

Comparison of endwall $\eta_{\text {eff }}$ for the CFD models with and without coolant plenums is shown in Figure 5(a). The results agree closely. This shows that decoupling the plenum has little effect on the flow solution.

Equations for scalar concentrations $\phi_{1}$ to $\phi_{N}$ were set up using the Fluent user-defined scalar feature. $N$ is the number of cooling holes. The following scalar boundary conditions were set at the inlet to hole $j$

$$
\begin{aligned}
& \phi_{i}=1, \text { for } i=j \\
& \phi_{i}=0, \text { for } i \neq j
\end{aligned}
$$

where the index $i$ denotes the scalar variable number and index $j$ denotes the cooling hole number.

The boundary condition $\phi_{i}=0$, for all $i$, is set at the freestream inlet. For each scalar variable, the scalar diffusivity was set to $\left(k+\left(\frac{c_{p} \mu_{T}}{\mathrm{Pr}_{\mathrm{t}}}\right)\right)$ so that the scalar diffusion is the same as the thermal diffusion (see equation (1)). This was done using a Fluent user-defined function.

The field equations for $\phi_{i}$ (equation (1)) were then solved. Equation (8) predicts the equality of $\eta_{\text {eff }}$ and $\Sigma \phi_{i}$ in the incompressible situation. This is confirmed in the plots in Figure 5(b): the lines for $\eta_{\text {eff }}$ and $\Sigma \phi_{i}$ agree extremely well, demonstrating the numerical consistency of the passive-scalar tracking method.

\section{Research objectives}

The purpose of the research is two-fold. Firstly, to demonstrate the novel scalar tracking method in (a)

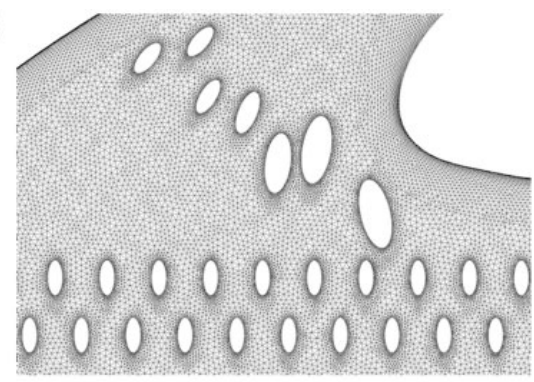

(b)

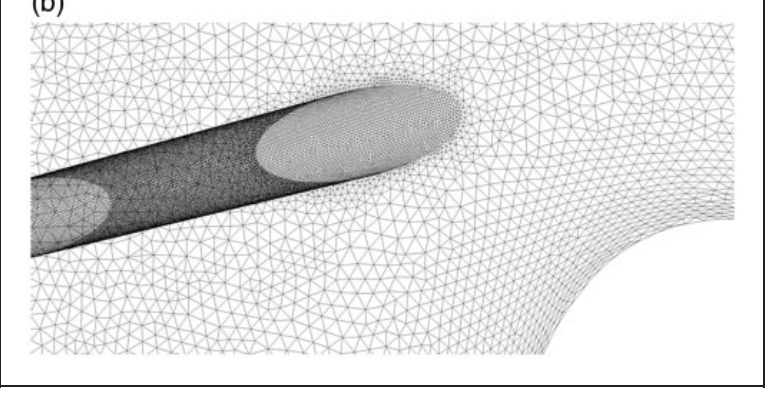

Figure 3. Endwall mesh resolution: (a) mesh on cooled endwall; (b) closeup of cooling hole mesh.

application to the optimisation of a nozzle guide vane endwall film cooling system. Secondly, using the new method, to develop an optimised cooling system that targets a minimum allowable value of platform film effectiveness with reduced cooling flow over a baseline design.

The optimum endwall film cooling system will deliver a target adiabatic cooling effectiveness, $\eta_{e f f}$, while using minimum coolant flow.

\section{Application to endwall cooling system optimisation}

The use of the passive-scalar tracking method will now be demonstrated by optimising cooling systems for a nozzle guide vane endwall. The physics of flow in the near-wall region of the vane is described briefly. The scalar tracking method is then used to re-design cooling systems to optimise distribution of the coolant flow.

\section{Background: Endwall secondary flows}

Flow through a turbine vane is generally well-behaved (no separations) because of strong acceleration and a favourable pressure gradient. Several authors describe the platform flows as dominated by secondary flows, however, as confirmed by smoke and oil flow visualisation experiments in linear cascades. Such studies are the basis of secondary flow models proposed by Langston et al., ${ }^{3}$ Sieverding and Van den Bosch, ${ }^{4}$ Sharma and Butler, ${ }^{5}$ and Wang and Olson. ${ }^{6}$

Figure 6(a) summarises important features of the models, which have the following common features: 


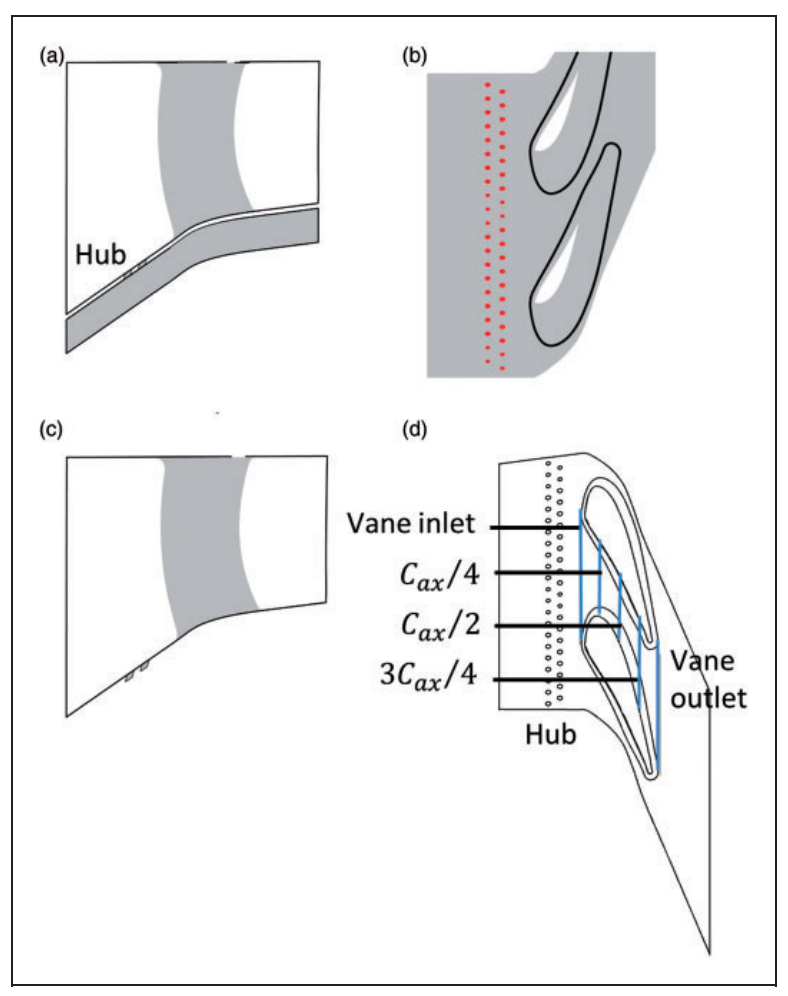

Figure 4. Passive-scalar tracking method implementation: (a) model with coolant plenum; (b) hole inlet boundaries; (c) model without coolant plenum; (d) postprocessing lines.

- Horseshoe vortex system. A horseshoe vortex is initiated upstream of the vanes and is composed of a pressure side and a suction side leg. The two legs interact in the downstream passage to strengthen the passage vortex.

- Boundary layer separation. The horseshoe vortex causes boundary layer separation, downstream of which a fresh boundary layer is formed.

- Cross-passage flow. The cross-passage pressure gradient (from vane PS to SS) drives low-velocity flow in the boundary layer further towards the SS.

The basic mechanism of formation of crosspassage flows near the endwall is described by Lakshminarayana. ${ }^{7}$ In Figure 6(b), flow streamline A lies outside the boundary layer while streamline B is near the endwall. Static pressure is roughly constant through the thickness of a fully developed boundary layer. ${ }^{8}$ Thus, streamline A and B are subjected to the same cross-passage pressure gradient, which is defined by the radial equilibrium equation

$$
\frac{\partial P}{\partial n}=\rho_{A} \frac{V_{A}^{2}}{R_{A}}=\rho_{B} \frac{V_{B}^{2}}{R_{B}}
$$

Since $V_{A}>V_{B}$, this implies $R_{A}>R_{B}$. i.e. streamline B has a smaller radius of curvature. This drives

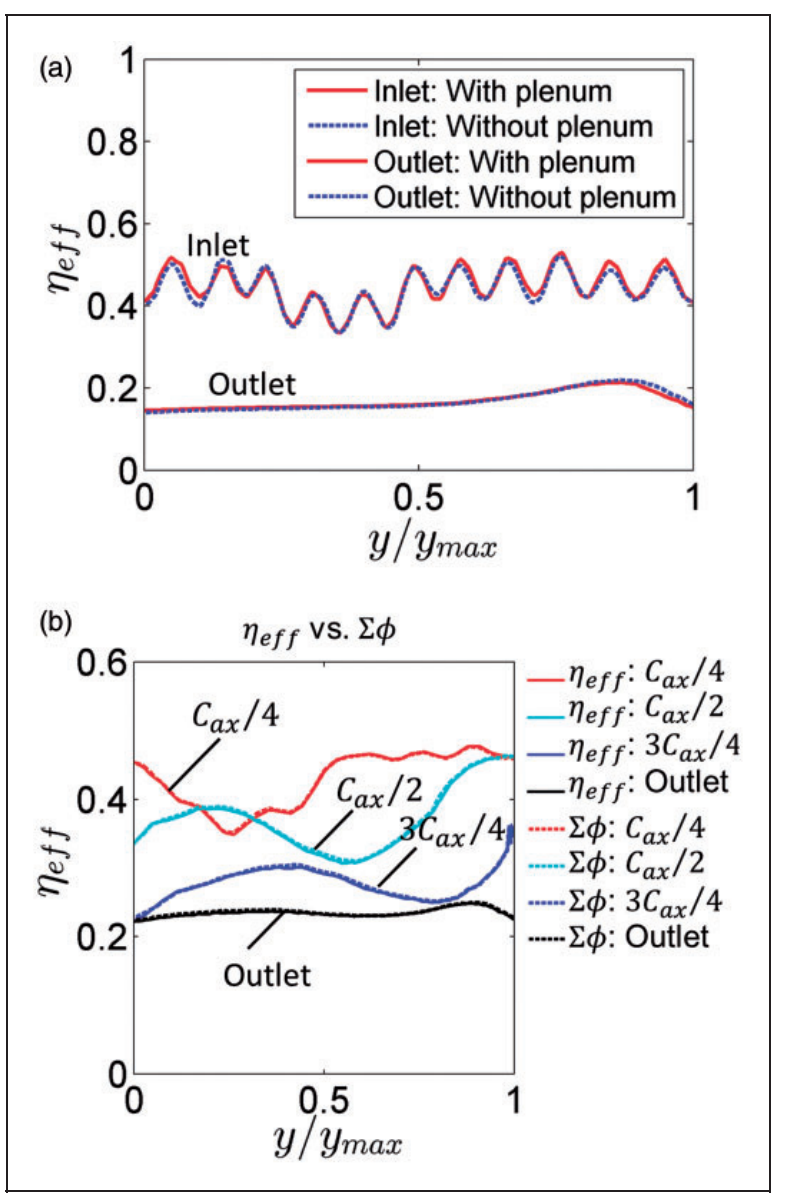

Figure 5. Passive-scalar tracking method: validation: (a) effect of decoupling plenum; (b) accuracy of scalar tracing method.

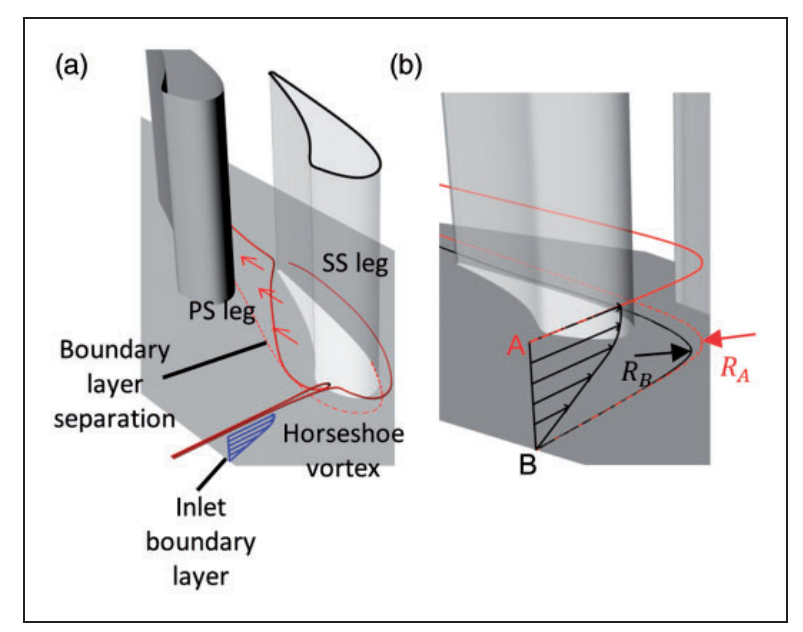

Figure 6. Classical secondary flow model: (a) schematic; (b) cross-passage flow mechanism.

near-wall flow towards the SS, resulting in a crosspassage flow.

Injecting high momentum flow into the boundary layer is known to suppress separation through reenergisation of the low momentum near-wall region. Sieverding and Wilputte ${ }^{9}$ measured flows in a vane 
passage with multiple cooling hole rows at compressible Mach numbers $(M=0.6,0.8)$. On injection of coolant, reduced cross-passage flows and a diminished passage vortex were observed. Granser and Schulenberg ${ }^{10}$ performed oil flow visualisation on two endwalls, one without film cooling and another with an upstream slot. At high slot momentum flux ratios, the horseshoe vortex separation line moved closer to the leading edge. Cross-passage flows were also reduced, because of the higher flow momentum in the near-wall region (see equation (12)). Thrift et al. ${ }^{11}$ obtained endwall cooling effectiveness data and velocity vector data in the vane stagnation plane. They demonstrate that the injection of streamwise momentum by a slot can accelerate the boundary layer and suppress horseshoe vortex formation. This effect is observed at high slot momentum flux ratio and low slot inclination.

Thomas et al. ${ }^{1}$ studied a double-row endwall cooling system with holes upstream of the vanes. Experiments and CFD were used to show that coolant injection altered near-wall flow patterns. Near-wall total pressure slightly exceeding mainstream total pressure resulted in suppression of endwall boundary layer separation and cross-passage flows. A large surplus in total pressure resulted in upwash on the vanes, moving coolant away from the endwalls, resulting in inefficient endwall cooling.

\section{Endwall cooling requirements}

In general, the trailing part of a HP vane endwall experiences the highest heat transfer rates, and is therefore the life-limiting region. This is because of high Mach numbers and thin boundary layers. ${ }^{13}$ The vane wake can have high turbulence levels, encouraging mixing and further heat transfer enhancement. In addition, the trailing part of the endwall can be difficult to cool. Films released in upstream low $M$ regions generally have low effectiveness on the trailing part of the vane, and local injection can result in high aerodynamic losses because of high freestream $M$.

\section{Application I: Targeting uniform $\eta_{\text {eff }}$ distribution}

Figure 7(a) shows a CFD model of the cooled hub endwall. A double row of cooling holes of uniform diameter are located upstream of the vane. This cooling system design is labelled Baseline1. Details of cooling hole geometry are given in Table 2. Flow solutions were obtained at incompressible conditions for an engine representative coolant mass flux ratio, $\dot{m}_{c} / \dot{m}_{\infty}=3.0 \%$.

Figure 7(c) shows $\eta_{\text {eff }}$ contours on the hub endwall cooled by the Baselinel design. The $\eta_{\text {eff }}$ distribution at the trailing endwall is non-uniform, with the coolant emerging as a streak at the PS TE. The pitchwise average of the near-wall total pressure at the vane inlet plane, marked in Figure 7(a), is shown in Figure 7(b). Coolant injection at high blowing ratio results in surplus near-wall $P_{0}$, which drives coolant migration towards the vane PS. The passive-scalar tracking method will now be used to target a uniform $\eta_{\text {eff }}$ distribution at the vane outlet line.

The scalar concentration equations (equation (1)) were solved for all 42 cooling holes in the domain. Scalar concentrations, $\phi_{i}$, on the vane outlet line are plotted in Figure 8(b). Holes marked 13 to 24 (see Figure 8(a)) were identified as significant contributors to cooling effectiveness. The contour plots for the scalar linked to hole $13\left(\phi_{13}\right)$ and hole $24\left(\phi_{24}\right)$ are shown in Figure 8(a). They show coolant emerging from the SS TE and PS TE, respectively. The vane

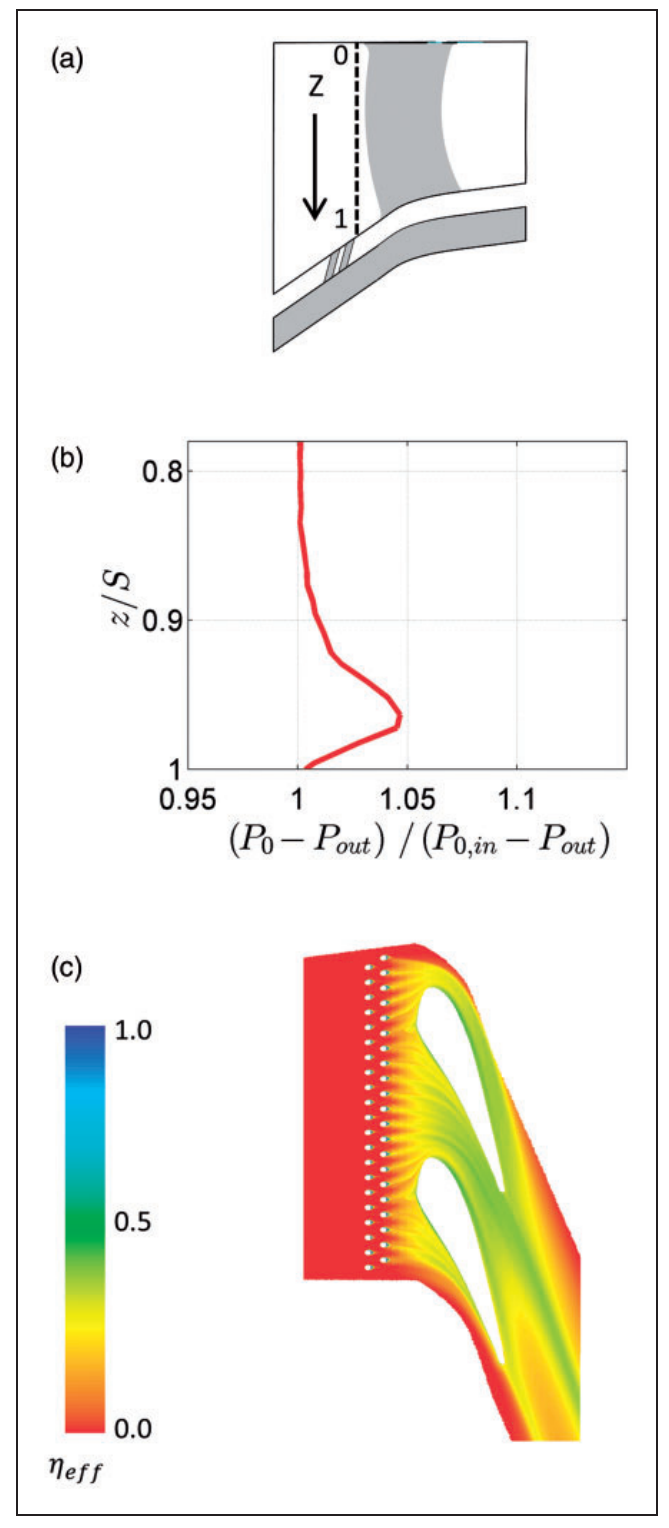

Figure 7. Cross-passage flow caused by coolant injection: (a) CFD model: Baselinel; (b) vane inlet total pressure profile; (c) Baselinel: $\eta_{\text {eff }}$ contours. 


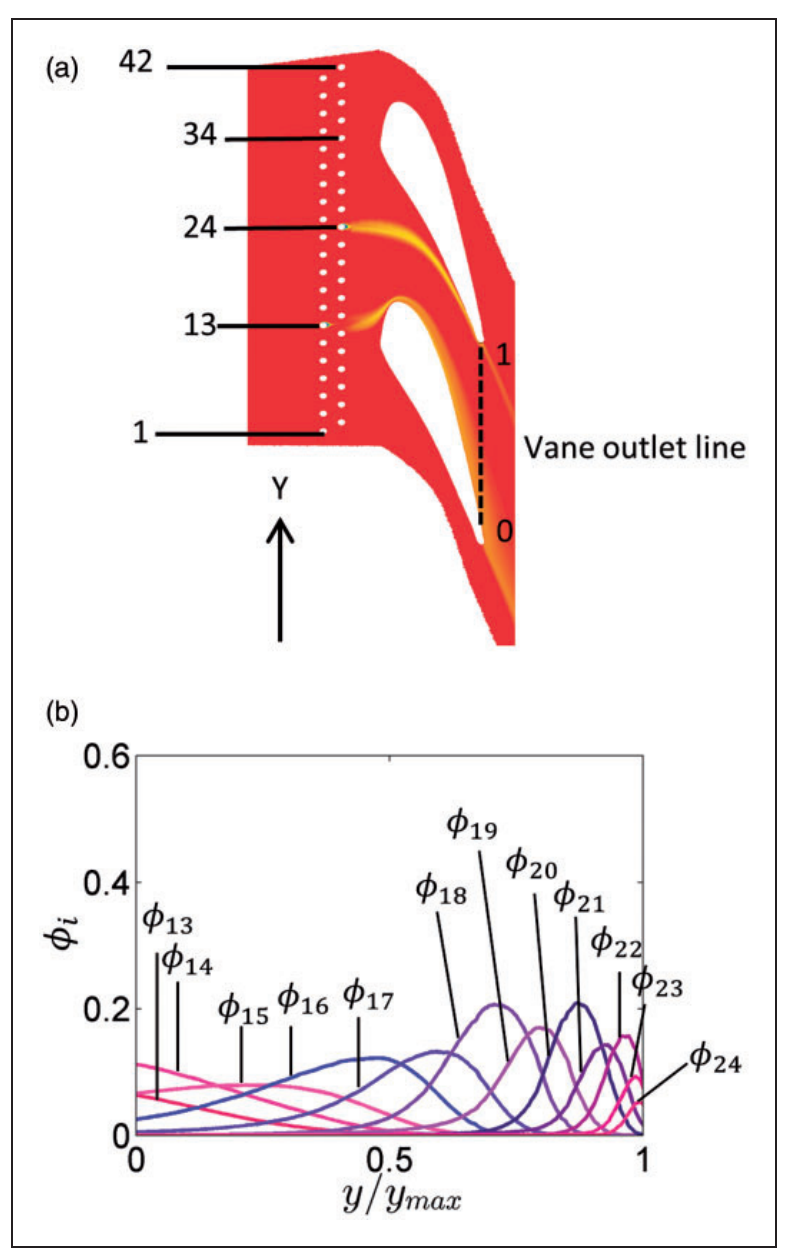

Figure 8. Scalar tracking method applied to hub cooling system: (a) contours of $\phi_{13}$ and $\phi_{24}$; (b) plots of $\phi_{i}$ at vane outlet.

outlet line, marked in Figure 8(a), spans the region cooled by these holes. Holes 13 to 34 cover one pitch length. The surplus near-wall total pressure (Figure 7(b)) drives upwash on the vanes, which carries coolant from holes 24 to 34 away from the endwall and into the freestream. Holes 24 to 34 thus do not contribute to cooling at the vane outlet. It can be seen from Figure 8(b) that coolant from holes 17 to 24 reaches $y / y_{\max }=0.5$ to 1.0 . Only coolant from holes 13 to 16 reaches $y / y_{\max }=0$ to 0.5 . The non-uniform distribution of coolant is caused by the cross-passage flow driving near-wall flow towards the vane PS.

The scalar tracking method allowed isolation of the holes that were key contributors to cooling effectiveness (holes 13 to 24). This distribution of coolant is now improved by adjusting the diameters of these holes.

A linear variation of diameters was prescribed for holes 13 to 24 . For hole number $j(13 \leqslant j \leqslant 24)$, the diameter $D_{j}$ is given by

$$
D_{j}=D_{13}+\frac{j-13}{24-13}\left(D_{24}-D_{13}\right)
$$

where $D_{13}$ and $D_{24}$ are hole diameters for $j=13$ and $j=24$ respectively. $D_{\text {base }}$ is the uniform hole diameter of the Baselinel cooling system. The notation is summarised in Figure 9.

The mass flux through individual cooling holes was obtained from the CFD solution. The variation in mass flux across holes 1-42 was less than $1 \%$ - the holes in Baselinel were sufficiently upstream of the vane that potential field effects were insignificant. Thus, for the Baseline1 design the total coolant flow for holes 1 to $N$ can be expressed as

$$
\dot{m}_{\text {original }}=k \sum_{j=13}^{24} D_{\text {base }}^{2}=k(24-13) D_{\text {base }}^{2}
$$

where $k$ is a constant. For small changes in hole diameters, the coolant flow rate through holes 13 to 24 can be approximated as

$$
\begin{aligned}
\dot{m}_{\text {new }} & =k \sum_{j=1}^{N} D_{j}^{2} \\
& =k \sum_{j=1}^{N}\left(D_{13}+\frac{(j-13)}{24-13}\left(D_{24}-D_{13}\right)\right)^{2}
\end{aligned}
$$

Coolant mass flow consumption is to remain unchanged. Setting $\dot{m}_{\text {original }}=\dot{m}_{\text {new }}$, and substituting values $D_{\text {base }}=6.9 \mathrm{~mm}$, the following expression was obtained

$$
D_{24}=\sqrt{135.49-0.81 D_{13}^{2}}-0.44 D_{13}
$$

For a particular value of $D_{13}$, the constant coolant mass flow requirement constrains $D_{24}$ to the value specified by equation (16). For small changes in diameter, $\eta_{\text {eff, new }}$ can be estimated using the superposition method of equation (10). This is repeated below

$$
\eta_{\text {eff, new }}=\sum_{j=1}^{42} \frac{D_{j, \text { new }}^{2}}{D_{\text {base }}^{2}} \phi_{j}
$$

Since $D_{j, \text { new }}=D_{\text {base }}$ for all holes besides $j=13$ to 24 , equation (17) can be rewritten as

$$
\eta_{\text {eff, new }}=\sum_{j=1}^{42} \phi_{j}+\sum_{j=13}^{24}\left(\frac{D_{j}^{2}}{D_{\text {base }}^{2}}-1\right) \phi_{j}
$$

$\eta_{\text {eff,new }}$ at the vane outlet line (marked in Figure 8(a)) is plotted for various values of $D_{13}$ in Figure 10(a). As required by equation (18), the plots for $\sum_{i=1}^{42} \phi_{i}$ and $D_{13}=D_{\text {base }}=6.9 \mathrm{~mm}$ overlay. This corresponds to the Baseline1 design. As $D_{13}$ increases, $\eta_{\text {eff, new }}$ increases at the vane outlet SS, and decreases 


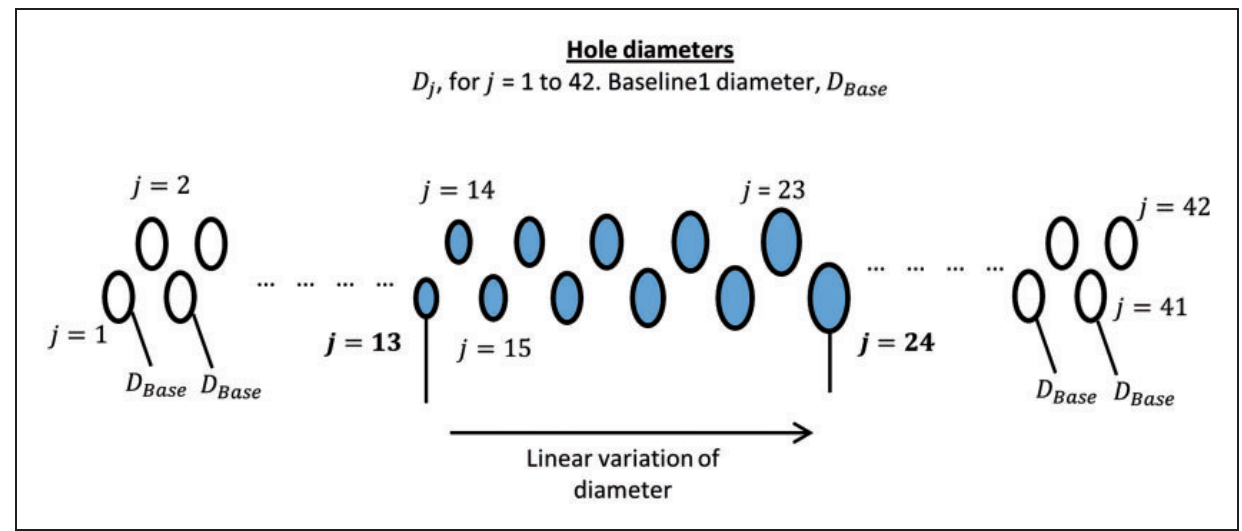

Figure 9. Hole diameter distribution.

at the vane outlet PS. The $\eta_{\text {eff, new }}$ distribution for $D_{13}=8.1 \mathrm{~mm}$ is considerably more uniform than the baseline design. The hole pattern for $D_{13}=8.1 \mathrm{~mm}$, called Config1, is shown in Figure 10(b). Diameters decrease linearly from hole $13\left(D_{13}=8.1 \mathrm{~mm}\right)$ to hole $24\left(D_{24}=5.5 \mathrm{~mm}\right)$.

A CFD model was solved for the Config1 hole pattern. Coolant flow rate was set to the Baseline1 condition $\left(\dot{m}_{c} / \dot{m}_{\infty}=3 \%\right)$. $\eta_{e f f}$ distributions for the Baseline1 and Configl designs are compared at the vane outlet in Figure 11(b). The $\eta_{\text {eff }}$ distribution is considerably more uniform for the Configl design than the Baseline1 design.

CFD predicted $\eta_{\text {eff }}$ contours for the Config1 design are shown in Figure 11(a). Coolant migrates to the PS TE in the Baselinel design (Figure 7(c)). This tendency remains unchanged with the Config1 hole pattern, but more coolant is introduced upstream of the vane SS than the vane PS, resulting in a more uniform $\eta_{\text {eff }}$ distribution at the vane outlet.

For the Configl design, the estimated cooling effectiveness $\left(\eta_{\text {eff,new }}\right.$ from equation (18)), is compared with the CFD result in Figure 11(b). The estimated change is of the correct sign and of approximately correct magnitude, but large changes in hole diameter lead to second-order effects, which are not accounted for in this approximate predictive approach. The discharge coefficient and initial entrainment of hot gas, for example, are both weak functions of hole diameter. An iterative approach would lead to more accurate predictions.

\section{Application 2: Reducing coolant consumption}

The cooling system for the casing endwall is now considered. $\eta_{\text {eff }}$ contours (CFD) on the casing endwall are shown in Figure 12(a). The cooling system (called Baseline2) consists of a double row of cooling holes of diameter, $D_{\text {base }}=8 \mathrm{~mm}$. Geometric details of this cooling system are given in Table 2 . A large amount of coolant $\left(\dot{m}_{c} / \dot{m}_{\infty}=6 \%\right)$ is injected through these holes, upstream of the vane

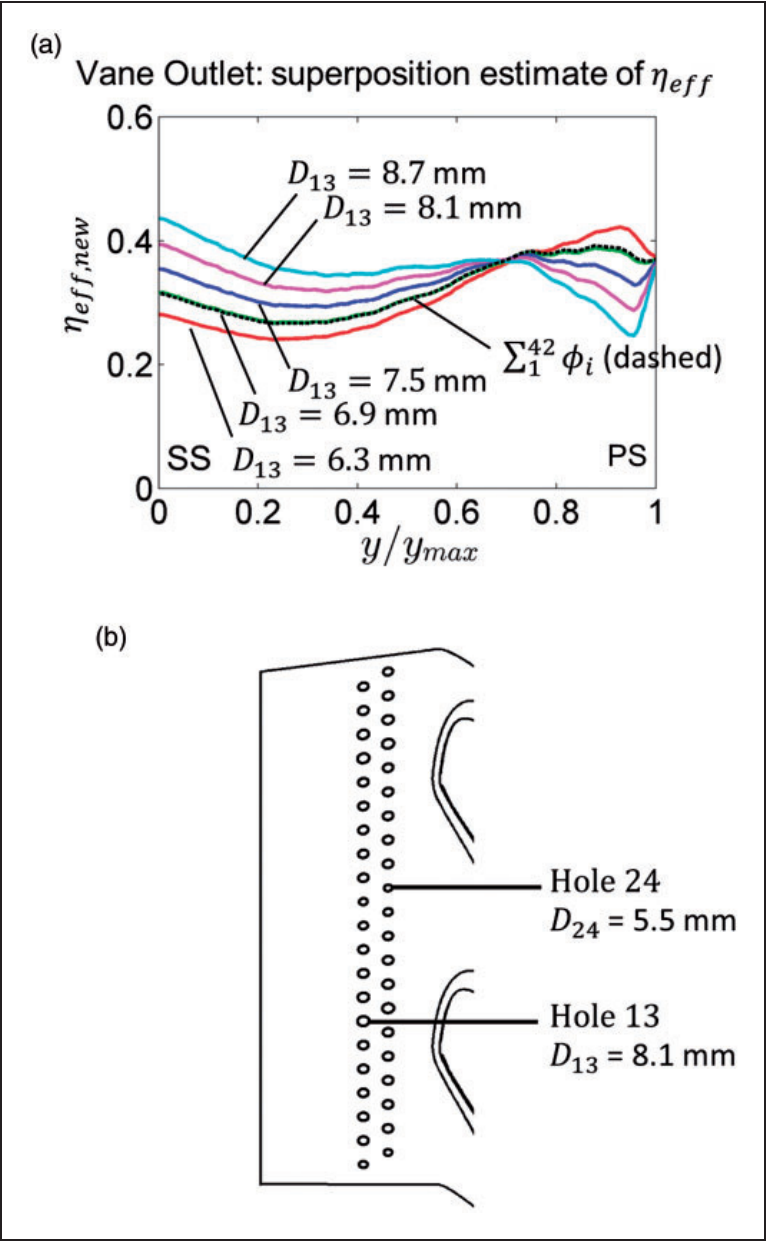

Figure 10. Optimisation using scalar tracking method:

(a) $\eta_{\text {eff, new }}$ estimated by superposition method for different $D_{13}$; (b) Config I: hole pattern.

passage. The injection of momentum at this location is beneficial: it suppresses endwall boundary layer separation and thus improves endwall cooling. However, the leading endwall is overcooled. As described previously, it is more important to cool the trailing endwall. The cooling system is now redesigned to reduce coolant consumption while maintaining similar levels of cooling effectiveness at the trailing endwall. 


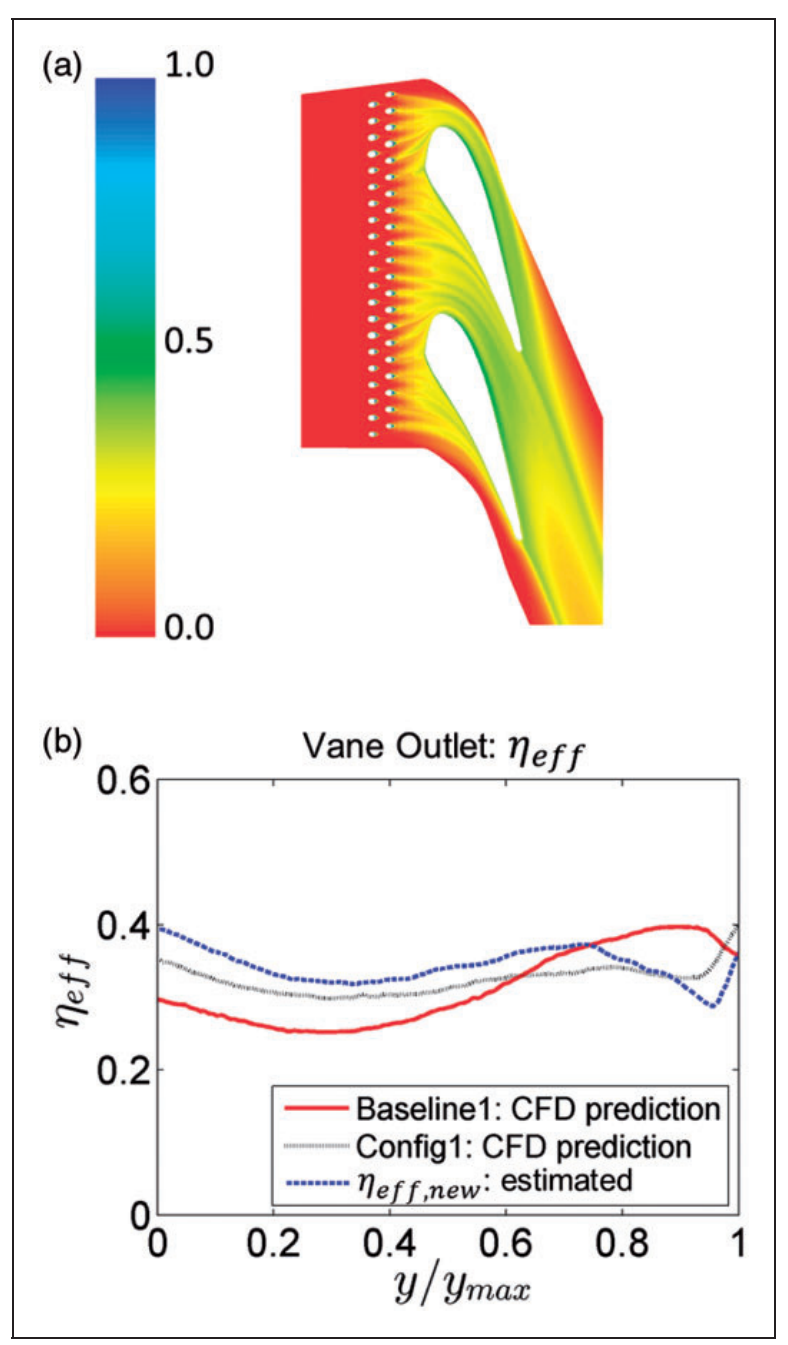

Figure II. Optimised hub cooling system: (a) ConfigI: $\eta_{\text {eff }}$ contours predicted by CFD; (b) ConfigI vs. Baselinel design.

To reduce coolant consumption, hole diameters were set to $0.5 D_{\text {baseline. The hole inclination was }}$ reduced from the baseline value of $40^{\circ}$, to $25^{\circ}$, a typical manufacturing limit. Lower hole inclinations suppress endwall boundary layer separation better. This is because of greater streamwise momentum injected into the boundary layer. Also lower hole inclinations result in improved $\eta_{\text {eff }}$ downstream.

A CFD simulation of this new cooling hole pattern was run with the same coolant total pressure, $P_{0, c}$. Surface streamlines were obtained. These are shown in Figure 12(b). New cooling holes, numbered 1-7, were placed mid-passage. These were aligned with the local surface streamline direction. Holes 1-3 direct coolant towards the SS endwall, while holes 5-7 direct coolant towards the PS endwall. Hole diameters were set to a constant value, $0.4 D_{\text {base }}$. Hole inclinations were set to $25^{\circ}$.

These simulations were performed at incompressible conditions. However, to demonstrate a procedure for optimisation, contours of isentropic Mach number were obtained from a compressible CFD simulation. All cooling holes were placed upstream of the $M=0.3$

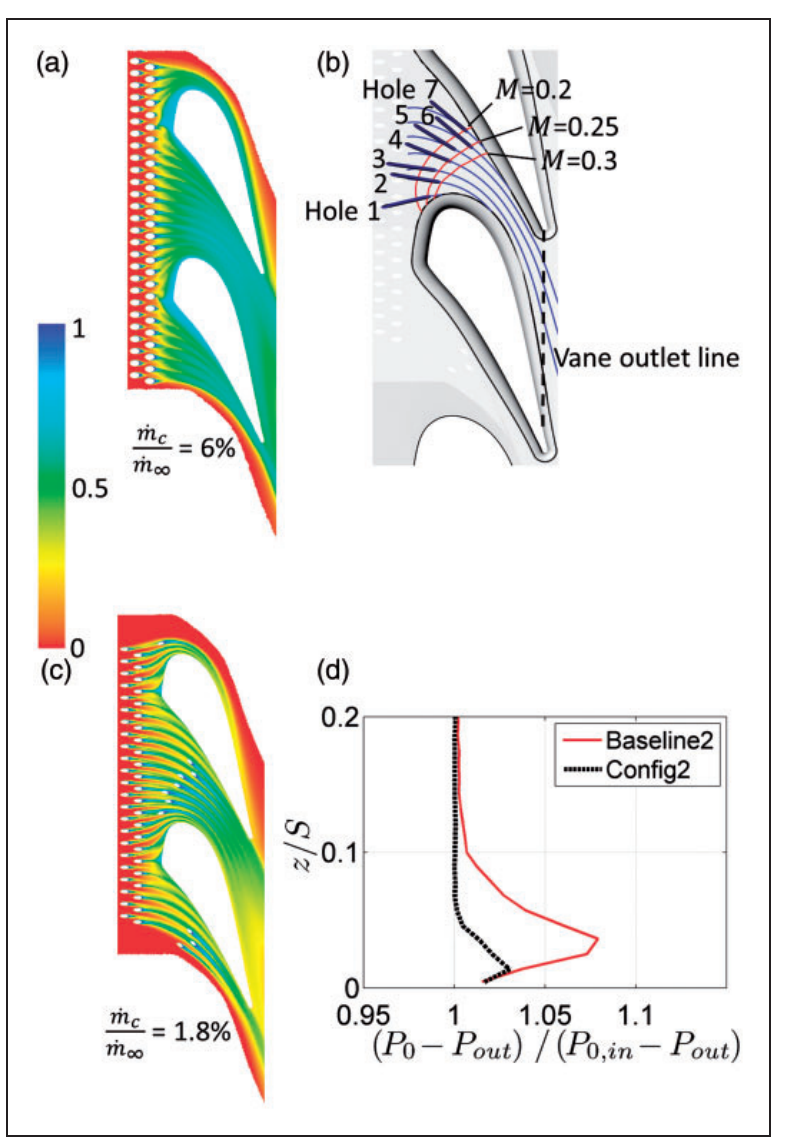

Figure 12. Optimisation procedure: Config2 design: (a) Baseline $2 \eta_{\text {eff }}$ contours; (b) mid-passage hole placement; (c) Config $2 \eta_{\text {eff }}$ contours and (d) near-wall total pressure profile.

contour (Figure 12(b)). This would result in minimal aerodynamic loss at engine condition. This choice of maximum Mach number for coolant injection was justified by Thomas et al. ${ }^{1}$ using the Kollen and Koschel $^{12}$ aerodynamic loss model.

The new cooling system design is labelled Config2. A CFD simulation was run with the same coolant total pressure, $P_{0, c}$. $\eta_{\text {eff }}$ contours for Config2 are shown in Figure 12(c). Endwall boundary layer separation does not occur upstream of the vane. The vane inlet $\hat{P}$ profile for the Baseline2 and Config2 design are shown in Figure 12(d). While reducing cooling hole diameters has decreased the near-wall total pressure peak, it retains a surplus over the freestream. This is able to suppress boundary layer separation, and drive passage cross-flows in the SS to PS direction. The reduction in near-wall total pressure will also reduce upwash on the vanes, and will keep coolant films near the endwall. The coolant consumption has decreased to $\dot{m}_{c} / \dot{m}_{\infty}=1.8 \%$ from $6 \%$. However cooling effectiveness at the trailing endwall has also decreased. The diameter of the mid-passage holes will now be adjusted to improve $\eta_{\text {eff }}$ at the vane outlet line.

Following the procedure described previously, scalar concentration equations were solved for the 


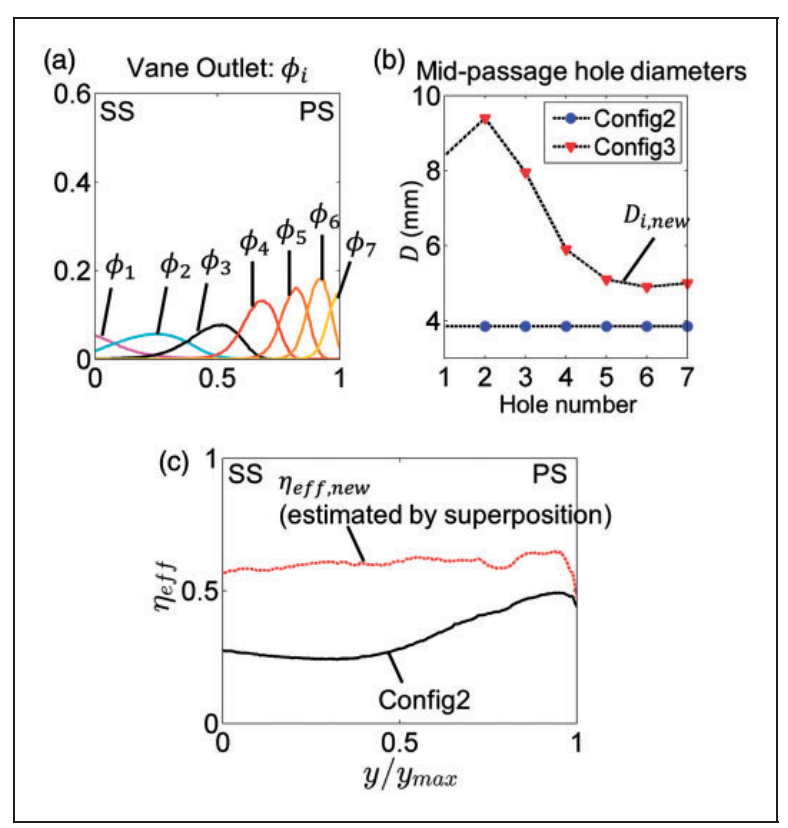

Figure 13. Optimisation of Config2 design using scalar tracking method: (a) scalar concentration plots; (b) final diameter distribution; (c) $\eta_{\text {eff }}$ distribution at vane outlet.

mid-passage holes. Scalar concentrations $\phi_{1}$ to $\phi_{7}$ correspond to holes 1 to 7 (marked in Figure 12(b)). Plots of $\phi_{1}$ to $\phi_{7}$ at the vane outlet line are shown in Figure 13(a).

Equation (10) was used to estimate $\eta_{\text {eff,new }}$ for new hole diameter distributions $\left(D_{i, \text { new }}\right)$, given $\phi_{1}$ to $\phi_{7}$. Total coolant mass flow rate was estimated using the expression

$$
\dot{m}_{c, \text { new }}=\dot{m}_{\text {Config } 2}+\sum_{i=1}^{7}\left(\frac{D_{i, \text { new }}^{2}}{D_{i, \text { base }}^{2}}-1\right) \dot{m}_{c, i}
$$

where $\dot{m}_{\text {Config } 2}$ was the coolant consumption of the Config2 design and $\dot{m}_{c, i}$ was the coolant flow rate through the mid-passage holes of Config2.

A uniform value of $\eta_{\text {eff }}=0.6$ was targeted at the vane outlet line (marked in Figure 12(b)). To achieve this goal, the diameters of holes 1-7 were adjusted manually until the predicted adiabatic effectiveness of the new design $\left(\eta_{\text {eff, new }}\right)$ was approximately 0.6 . The final diameter distribution and $\eta_{\text {eff,new }}$ are shown in Figure 13(b) and (c). The total coolant mass flux ratio (mid-passage and upstream holes) estimated using equation (19) was $\dot{m}_{c} / \dot{m}_{\infty}=2.9 \%$.

A CFD simulation was run with this new distribution of mid-passage hole diameters. The design, called Config3, is shown in Figure 14(a). Flow boundary conditions, including coolant total pressure, were not changed. The endwall $\eta_{\text {eff }}$ contours for Config3 are shown in Figure 14(b). The CFD predicted coolant mass flux ratio for the Config3 design was $\dot{m}_{c} / \dot{m}_{\infty}=3.0 \%$, closely matching the value estimated by equation (19), $\dot{m}_{c} / \dot{m}_{\infty}=2.9 \%$.

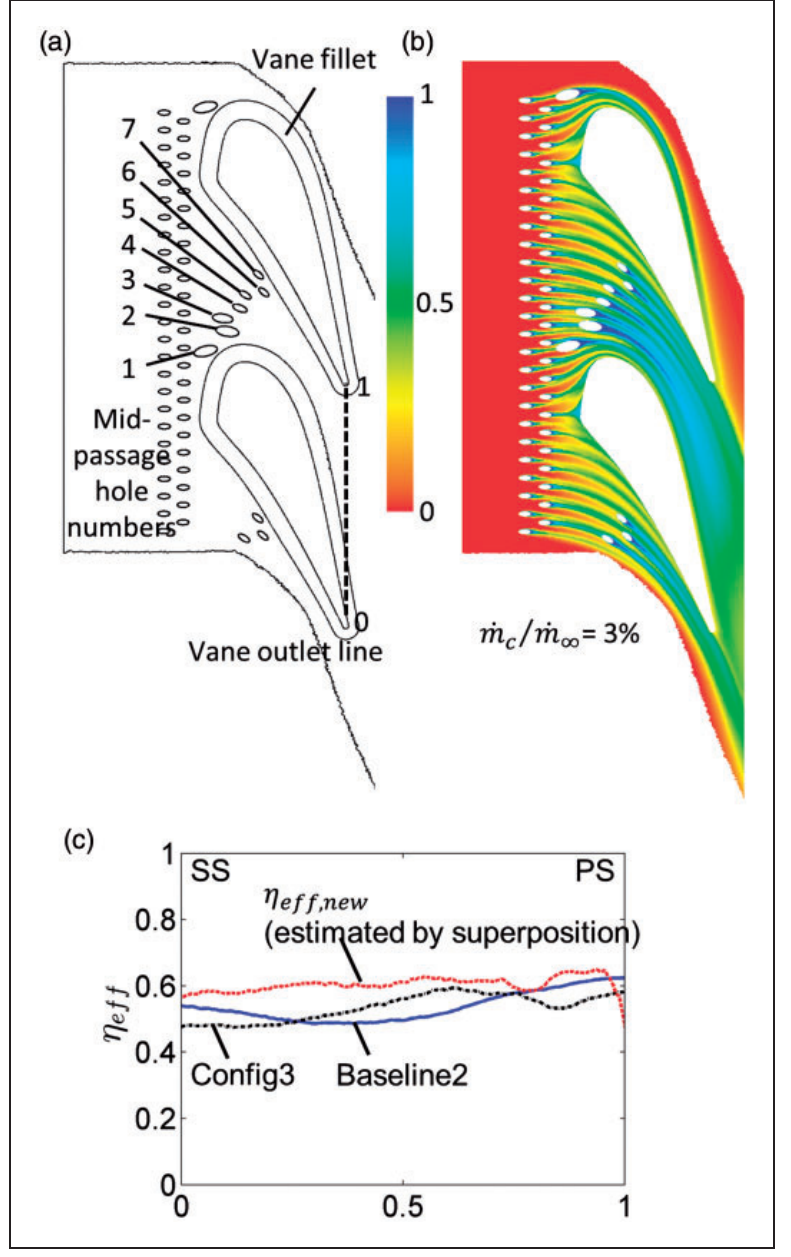

Figure 14. Config3 design: (a) Config3 design schematic; (b) Config3: $\eta_{\text {eff }}$ contours; (c) $\eta_{\text {eff }}$ distribution at vane outlet.

The $\eta_{\text {eff }}$ distributions for the Baseline 2 and Config3 designs are compared at the vane outlet in Figure 14(c). While coolant consumption for the Config3 design has decreased by $50 \%$ from the Baseline 2 design, the mean $\eta_{\text {eff }}$ at vane outlet is only $5 \%$ lower. The Config3 design has thus achieved the optimisation goal of reducing coolant consumption while maintaining similar levels of cooling effectiveness on the trailing endwall.

Figure 14(c) also compares $\eta_{\text {eff,new }}$ estimated by equation (10), with the CFD prediction (marked Config3). The mismatch is small despite the large adjustments in diameter. The target $\eta_{\text {eff }}$ of 0.6 could be achieved with greater accuracy if this process were iterated using scalar concentration distributions for the Config3 solution.

$\eta_{\text {eff }}$ contours on the vane for the Baseline 2 and Config3 design are shown in Figure 15(a) and (b). The $\eta_{\text {eff }}$ distributions on a spanwise line are compared in Figure 15(c). Coolant is driven up the vane surface and away from the endwall in the Baseline 2 design. The vane inlet $\hat{P}$ profiles for the Baseline 2 and Config2 design were shown in Figure 12(d). Since the upstream cooling hole rows have not been 


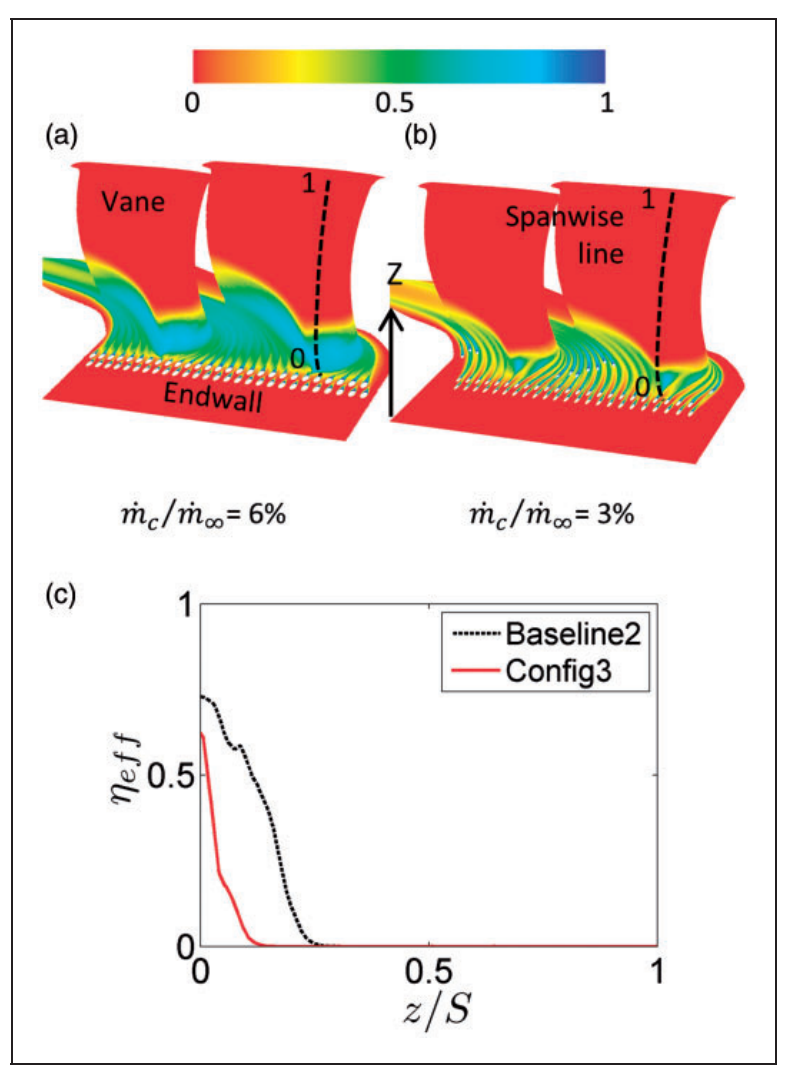

Figure 15. Coolant upwash on vane: (a) Baseline2 design: $\eta_{\text {eff }}$ contours; (b) Config3 design: $\eta_{\text {eff }}$ contours; (c) spanwise plot of $\eta_{\text {eff }}$

modified, the $\hat{P}$ profiles for the Config2 and Config3 designs are approximately the same. Coolant injection leads to surplus total pressure in the near-wall region - this drives upwash on the vane surface. The peak in $\hat{P}$ is closer to the endwall in the Config3 design than the Baseline2 design because of lower hole inclination. The near-wall $\hat{P}$ values are also smaller because of lower coolant flow rate. This results in reduced upwash and more efficient cooling of the endwall.

\section{Conclusions}

A novel passive-scalar tracking method has been presented which isolates cooling effectiveness contributions from individual cooling holes. The purpose of the method is to allow accurate cooling superposition techniques in complex film cooled environments. This in turn allows a rapid iterative convergence on optimum designs, without the need to search a wide design space. The technique is powerful and results in order of magnitude reductions in the number of simulations necessary to achieve an optimum design - the additional computational cost to solve the passive-scalar equations is modest in comparison to the saving achieved.

The scalar tracking method has been implemented in CFD, and its mathematical accuracy verified. To demonstrate the technique in application, the method was used to optimise a nozzle guide vane endwall cooling system. Highly optimised film cooling arrangements were achieved, with effectiveness distributions close to a target profile after a small number of simulations. Coolant reduction for the systems analysed was approximately $50 \%$ for similar limiting effectiveness values.

The passive-scalar tracking method was shown to be an accurate and effective tool for optimising film cooling systems using CFD. Using this method, an optimised film cooling system can be designed with only three to five CFD runs. This is an order of magnitude less computationally expensive than optimisation performed using automated design space exploration.

It is advocated that designers adopt the method for further optimisation studies of this type.

\section{Acknowledgments}

The authors gratefully acknowledge Rolls-Royce plc. for project funding and technical contributions.

\section{Conflict of interest}

The authors received no financial support for the research, authorship, and/or publication of this article.

\section{Funding}

This project was jointly funded by the Engineering and Physical Sciences Research Council (EPSRC) and RollsRoyce plc.

\section{References}

1. Thomas M, Kirollos B, Jackson D, et al. Experimental and CFD studies of NGV endwall cooling. ASME paper no. GT2013-95639, 2013.

2. Bradshaw P. Turbulence modeling with application to turbomachinery. Prog Aerosp Sci 1996.

3. Langston LS, Nice ML and Hooper RM. Three-dimensional flow within a turbine cascade passage. ASME J Eng Power 1977; 99(1): 21-28.

4. Sieverding $\mathrm{CH}$ and Van den Bosche P. The use of coloured smoke to visualize secondary flows in a turbine cascade. J Fluid Mech 1983; 134: 85-89.

5. Sharma OP and Butler TL. Predictions of endwall losses and secondary flows in axial flow turbine cascades. ASME J Turbomach 1987; 109(2): 229-236.

6. Wang HP, Olson SJ, Goldstein RJ, et al. Flow visualization in a linear turbine cascade of high performance turbine blades. ASME J Turbomach 1997; 119(1): 1-8.

7. Lakshminarayana B. Fluid dynamics and heat transfer of turbomachinery. New York: John Wiley \& Sons, 1996.

8. Schlichting H and Gersten K. Boundary layer theory. 8th ed. New York: Springer, 2000.

9. Sieverding $\mathrm{CH}$ and $\mathrm{Ph}$ Wilputte. Influence of Mach Number and End Wall Cooling on Secondary Flows in a Straight Nozzle Cascade. J Eng Gas Turb Power 1981; 103(2): 257-263.

10. Granser D and Schulenberg T. Prediction and measurement of film cooling effectiveness for a firststage turbine vane shroud. ASME paper no. 90GT-95, 1990. 
11. Thrift A, Thole $\mathrm{K}$ and Hada $\mathrm{S}$. Impact of the combustor-turbine interface slot orientation on the durability of a nozzle guide vane endwall. J Turbomach 2013.

12. Köllen $\mathrm{O}$ and Koschel W. Effect of film cooling on the aerodynamic performance of a turbine cascade. In: AGARD Heat Transfer and Cooling in Gas Turbines, Vol. 1, 1985, p.16.

13. Nicklas M. Film-cooled turbine endwall in a transonic flow field: Part II-heat transfer and film-cooling effectiveness. J Turbomach 2001; 123(4): 720-729.

\section{Appendix I}

\section{Notation}

$\begin{array}{ll}c_{p} & \text { specific heat }(\mathrm{J} / \mathrm{kg} / \mathrm{K}) \\ C_{a x} & \text { axial chord length }(\mathrm{m}) \\ D & \text { hole diameter }(\mathrm{m}) \\ I & \text { momentum flux ratio } \\ k & \text { turbulence kinetic energy }(\mathrm{J} / \mathrm{kg}) \\ l & \text { turbulence length scale }(\mathrm{m}) \\ L & \text { hole length }(\mathrm{m}) \\ \dot{m} & \text { mass flow rate }(\mathrm{kg} / \mathrm{s}) \\ M & \text { Mach number } \\ P & \text { pressure }(\mathrm{Pa}) \\ \hat{P} & \text { non-dimensional total pressure } \\ & \left(P_{0}-P_{\text {out }}\right) /\left(P_{0, \text { in }}-P_{\text {out }}\right) \\ P r & \text { Prandtl number } \\ R e & \text { Reynolds number } \\ S & \text { vane span }(\mathrm{m}) \\ T u & \text { turbulence intensity }(\%) \\ U & \text { velocity }(\mathrm{m} / \mathrm{s}) \\ V & \text { vane pitch }(\mathrm{m}) \\ X & \text { axial coordinate } \\ Y & \text { pitchwise coordinate } \\ Z & \text { spanwise coordinate } \\ \varepsilon & \text { turbulence dissipation rate }(\mathrm{J} / \mathrm{kg} / \mathrm{s}) \\ \eta_{e f f} & \text { adiabatic effectiveness } \\ \rho & \text { density }\left(\mathrm{kg} / \mathrm{m}^{3}\right) \\ \phi & \text { scalar concentration }\left(\mathrm{kg}{ }^{-1}\right) \\ & \end{array}$

\section{Subscripts}

$\begin{array}{ll}\text { aw } & \text { adiabatic wall } \\ \text { base } & \text { baseline design } \\ c & \text { coolant conditions } \\ e & \text { entrained flow } \\ \text { in } & \text { domain inlet conditions } \\ m & \text { mixed conditions } \\ \text { new } & \text { new design } \\ \text { out } & \text { domain outlet conditions } \\ r & \text { recovery conditions }\end{array}$

stagnation conditions

$\infty \quad$ freestream conditions

\section{Appendix 2}

\section{Use of scalar tracking method in compressible conditions}

In compressible flow, the adiabatic wall temperature, $T_{a w}$, is the local recovery temperature, $T_{r}$. This can be estimated from $T_{0, m}$ using the equation

$$
T_{a w}=T_{r}=T_{0, m}-(1-r) \frac{u_{\infty}^{2}}{2 c_{p}}
$$

where $r=\operatorname{Pr}^{1 / 3}$ is the recovery factor in turbulent flows, and $u_{\infty}$ is the local freestream velocity. Unlike the incompressible case, $T_{a w}$ is not equal to $T_{0, m}$. Thus, the simplification of equation (8) is no longer accurate - that is, $\Sigma \phi_{i}$, is not an accurate proxy for $\eta_{\text {eff. }}$. To optimise cooling systems in compressible flows, it is simpler to work with $T_{0, m}$ as a target, where $T_{0, m}$ is estimated from $\Sigma \phi_{i}$ using the following equation

$$
T_{0, m}=T_{0, \infty}-\left(\sum_{i} \phi_{i}\right)\left(T_{0, \infty}-T_{0, c}\right)
$$

On the vane outlet line considered for optimisation in this paper, freestream velocity, $u_{\infty}$, is approximately constant. From equation (20), $T_{0, m}$ is offset from $T_{a w}$ by an approximate constant quantity. A uniform $T_{0, m}$ on the vane outlet line will, for example, yield an approximately uniform $T_{a w}$.

\section{Accounting for specific heat variation}

Specific heat $\left(c_{p}\right)$ varies with temperature. An improved derivation of the passive scalar tracking method is now provided, which accounts for this variation. The continuity and energy equations applied to Figure 1 are given below

$$
\begin{aligned}
& \dot{m}_{m}=\dot{m}_{e}+\dot{m}_{c} \\
& \dot{m}_{m} c_{p, m} T_{0, m}=\dot{m}_{e} c_{p, \infty} T_{0, \infty}+\dot{m}_{c} c_{p, c} T_{0, c}
\end{aligned}
$$

Dividing equation (23) by $\dot{m}_{m} c_{p, \infty}$, eliminating $\dot{m}_{e}$ using equation (22), and setting $\Sigma \phi=\dot{m}_{c} / \dot{m}_{m}$, the following equation was obtained

$$
\frac{c_{p, m}}{c_{p, \infty}} T_{0, m}=(1-\Sigma \phi) T_{0, \infty}+\Sigma \phi \frac{c_{p, c}}{c_{p, \infty}} T_{0, c}
$$


Assuming a linear variation of $c_{p}$ with temperature and setting $\kappa=c_{p, c} / c_{p, \infty}$ (constant), an expression for $c_{p, m} / c_{p, \infty}$ can be obtained

$$
\frac{c_{p, m}}{c_{p, \infty}}=1-(1-\kappa) \frac{T_{0, \infty}-T_{0, m}}{T_{0, \infty}-T_{0, c}}
$$

The expression for $c_{p, m} / c_{p, \infty}$ is substituted in equation (24) to obtain

$$
\begin{array}{r}
\left(1-(1-\kappa) \frac{T_{0, \infty}-T_{0, m}}{T_{0, \infty}-T_{0, c}}\right) T_{0, m} \\
=(1-\Sigma \phi) T_{0, \infty}+(\Sigma \phi) \kappa T_{0, c}
\end{array}
$$

This is a quadratic expression for $T_{0, m}$, which can be solved for given values of $T_{0, \infty}, T_{0, c}, \kappa$ and scalar concentration sum $\Sigma \phi$. The cooled adiabatic wall temperature $T_{a w}$ can be estimated from $T_{0, m}$ using equation (20).

For a typical gas turbine, $\kappa=c_{p, c} / c_{p, \infty}=0.9$ for $T_{0, c} \sim 900 \mathrm{~K}$ and $T_{0, \infty} \sim 1800 \mathrm{~K}$. Substituting $\kappa=0.9, \Sigma \phi=0.6$ in equation (26), $T_{0, m}$ is estimated as $1282 \mathrm{~K} . T_{0, m}$ estimated with $\kappa=1$ (specific heat invariant with temperature) is $1260 \mathrm{~K}$ : when constant $c_{p}$ is assumed, near-wall fluid temperature is underestimated by $22 \mathrm{~K}$. 